\title{
Electrodiagnosis in Amyotrophic Lateral Sclerosis
}

\author{
Nanette C Joyce, D.O., M.A.S. ${ }^{1}$ and Gregory T Carter, M.D., M.S. ${ }^{1,2}$ \\ ${ }^{1}$ Department of Physical Medicine and Rehabilitation, University of California, Davis School of \\ Medicine, 4860 Y Street Suite 3850, Sacramento, CA. 95817 \\ ${ }^{2}$ Department of Neurosciences, Physiatry Division, Providence Medical Group, 413 Lilly Road \\ Northeast, Olympia, WA 98506
}

\begin{abstract}
Electrophysiology remains an important tool in the evaluation of patients presenting with signs and symptoms of motor neuron disease (MND). The electrodiagnostic study should include peripheral nerve conduction studies (NCS) and needle electromyography (EMG) to both exclude treatable disease and gather evidence toward a diagnosis of amyotrophic lateral sclerosis (ALS). The recent changes in the revised El Escorial criteria, recommended by the Awaji-shima consensus group, have increased the diagnostic significance of fasciculation potentials to equal that of fibrillation and positive sharp wave potentials in the needle EMG examination of the patient suspected of having ALS. In addition, electrophysiologic evidence is now considered equivalent to clinical signs and symptoms in reaching diagnostic certainty of ALS. These changes, strategies for the design and implementation of an effective electrodiagnostic evaluation, and additional electrophysiologic techniques and their relationship to the evaluation of a patient with ALS are reviewed and discussed.
\end{abstract}

\section{Keywords}

amyotrophic lateral sclerosis; motor neuron disease; Lou Gehrig's disease; electrodiagnosis; electromyography

\section{Introduction}

Amyotrophic lateral sclerosis (ALS) is a progressive and inevitably fatal neurodegenerative disease belonging to a heterogeneous group of disorders known as motor neuron diseases. ALS is the most common adult MND worldwide and is considered the prototypical disorder of this class, as demonstrated by the often-interchangeable use of the terms ALS and MND. ${ }^{1}$ However, while ALS is characterized by the rapid degeneration of motor neurons throughout the neuraxis and by definition requires both upper motor neuron (UMN) and lower motor neuron (LMN) signs to confirm the clinical diagnosis, other motor neuron diseases such as spinal muscular atrophy (SMA) or the atypical motor neuron variants,

Publisher's Disclaimer: This is a PDF file of an unedited manuscript that has been accepted for publication. As a service to our customers we are providing this early version of the manuscript. The manuscript will undergo copyediting, typesetting, and review of the resulting proof before it is published in its final citable form. Please note that during the production process errors may be discovered which could affect the content, and all legal disclaimers that apply to the journal pertain. 
progressive muscular atrophy (PMA), primary lateral sclerosis(PLS), and progressive bulbar palsy (PBP), have more focal involvement or only UMN or LMN involvement, at least initially. ${ }^{2,3}$ Because prognosis and survival can be vastly different among the motor neuron disorders, differentiating among these disorders and making an accurate and timely diagnosis are paramount.

Individually, the motor neuron diseases are rare with the prevalence of ALS being approximately 5-7 per 100,000 people. ${ }^{4}$ Limited DNA analysis is available and can identify the etiology for some patients with familial ALS (10-15\% of all ALS patients), as well as those with SMA and spinal bulbar muscular atrophy (SBMA); but for the majority of patients with symptoms of MND, diagnosis is complicated by the lack of disease biomarkers. Thus, a process of exclusion is used to determine the diagnosis. Laboratory testing, neuroimaging, and muscle biopsy are all potentially helpful in focusing the differential diagnosis and excluding processes that mimic ALS (Table 1). Electrodiagnostic evaluation is key to establishing the diagnostic certainty of ALS once other disease processes have been excluded.

\section{General Approach to the Electrodiagnostic Evaluation of the Patient with} ALS

Clinically, ALS causes muscle atrophy and weakness. Symptoms most typically begin at one anatomic site and spread, with the disease progressing until the patient loses volitional control of skeletal muscles, transitioning from independence to dependence. In the late stages, mechanical ventilation and nutritional support may be required. The spread of symptoms is so typical of ALS that it is required for diagnosis. ${ }^{2,3}$

Signs at presentation may be limited to UMN or LMN loss. Phenotypic presentation can be markedly varied in ALS, making early diagnosis more complicated. Chio and colleagues examined the phenotypes of over 1300 ALS patients and described eight common presentations: Classic, bulbar, flail arm, flail leg, pyramidal, pure LMN, pure UMN, and respiratory. ${ }^{5}$ While late stage disease is relatively easy to identify, early disease can be much more difficult to diagnose on clinical examination alone. It is in this situation, early in disease when LMN abnormalities may be hidden from clinical appreciation, that electrodiagnostic evaluation becomes most valuable.

Electrodiagnostic testing is considered an extension of the physical examination and should be performed on all patients suspected of having a MND. The MND differential is divided between those with predominant UMN and/or LMN findings (Table 2). Assessing muscle atrophy and tone and testing for abnormal muscle stretch reflexes in all four anatomic regions - bulbar, cervical, thoracic, and lumbar - should necessarily be included in the physical examination and will inform the electrodiagnostic study. ${ }^{1-3,6}$ For example, a purely UMN disorder such as PLS with clinical signs of spasticity and hyperreflexia should result in a normal electrodiagnostic study. The various purely LMN disorders, with signs of muscle atrophy and hyporeflexia on examination, will share among them several electrodiagnostic abnormalities, detailed below. 
The electrodiagnostic evaluation in a suspected MND case requires peripheral NCS and needle EMG. Additional techniques such as repetitive stimulation, single fiber EMG, and motor unit number estimation (MUNE) have been used in ALS evaluation, and may be helpful in more challenging cases.

\section{Peripheral Nerve Conduction Studies in ALS}

The MND electrodiagnostic evaluation includes peripheral NCS examining at least one upper and one lower limb, and should begin by evaluating the most severely affected sites. ${ }^{1,6,7}$ A complete evaluation includes motor, sensory, and F-wave nerve conduction studies.

Motor conduction studies are an essential part of the electrodiagnostic evaluation of a patient suspected of having a MND. These studies allow the exclusion of treatable neuropathies, such as multifocal motor neuropathy with conduction block, from the differential diagnosis and should include proximal stimulation sites (above the elbow and Erb's point) to rule out conduction block and temporal dispersion, which are not typical findings in ALS. ${ }^{1,6-8}$ Motor NCS of the median, ulnar, and peroneal muscles should be evaluated.

Common findings observed from motor NCS in patients with MND include asymmetric side-to-side CMAP differences, normal CMAPs, or CMAPs with decreased amplitude, prolonged distal motor latency, and slowed conduction velocity consistent with axon loss. ${ }^{6-8}$ In a study comparing ulnar conduction in ALS to matched control subjects, a strong correlation was observed between CMAP amplitude and MRC grade strength ( $\mathrm{r}=0.81)$ : as strength decreased, CMAP amplitude decreased. ${ }^{9}$ CMAP amplitude was normal in all muscles with MRC 5 strength, even when the patient's contralateral limb was weak and had documented CMAP abnormalities. ${ }^{6-9}$ In addition, distal motor latencies and slowing of conduction velocity worsened as the severity of muscle weakness increased $(\mathrm{P}<0.001) .{ }^{9}$ These findings have been attributed to slowly conducting distal regenerating motor axons and loss of the fastest conducting lower motor neurons. ${ }^{8,10,11}$

Sensory conduction studies should include the ulnar and sural nerves; it is generally accepted that sensory nerves are normal in ALS. ${ }^{1,6,7,9}$ Consistent with this, no abnormalities were observed in the antidromic ulnar sensory conduction studies recorded from all 137 ulnar nerves of the ALS subjects in the above study. ${ }^{9}$ However, there are multiple reports in the literature documenting sensory abnormalities in patients with ALS. ${ }^{12-14}$ In a prospective study of 88 ALS patients, 20 subjects (22.7\%) had evidence of sensory nerve conduction abnormalities. ${ }^{15}$ The authors noted that this greatly surpassed the prevalence of polyneuropathy both in the background population as well as in the general population older than 55 years of age. They hypothesized that these results point to the existence of a subset of ALS patients who, in addition to their motor neuron pathology, have dorsal root ganglion degeneration. ${ }^{15}$ Findings of dorsal root ganglion abnormalities in SOD1 ALS mice supports this hypothesis. ${ }^{16}$ A multicenter prospective follow-up study demonstrated similar findings, with $17 \%$ of 35 patients showing abnormalities on sural sensory nerve conductions. ${ }^{17}$ The discovery of sensory abnormalities, while suggestive of a different disease process, do not completely exclude ALS. Other MNDs commonly associated with sensory nerve conduction 
abnormalities are spinobulbar muscular atrophy (sensory neuropathy) and hereditary spastic paraplegia (sensorimotor neuropathy). ${ }^{18,19}$

\section{F-Wave Studies}

Several studies have reported abnormalities in F waves of patients with ALS. ${ }^{8,20,21} \mathrm{~F}$-wave persistence when compared with controls was abnormal in 23 patients with MND and decreased as MRC graded strength decreased. F-wave latencies and chronodispersion, defined as the difference between the maximal and minimal F-wave latencies, were increased in the ALS patients as compared to the controls in this study by Argyriou and colleagues. 8

\section{Needle Electromyography in ALS}

Needle electromyography is the most important component of the electrodiagnostic evaluation in MND. It allows identification of LMN involvement often before it becomes clinically evident, extending the physical examination and enabling earlier diagnosis. ${ }^{1-3,6,7}$ For the evaluation of LMN findings in ALS, the clinical and electrophysiological abnormalities have equal diagnostic significance in any given body region. ${ }^{3}$ However, two EMG features are required for confirmation of neurogenic change consistent with a diagnosis of ALS:

- Evidence of chronic neurogenic change.

- $\quad$ Evidence of acute denervation. ${ }^{3}$

To support a diagnosis of ALS, the needle electrode examination should reveal decreased motor unit recruitment with rapid firing of a reduced number of motor units, and/or large amplitude, long duration MUP with or without evidence of remodeling (increased number of phases) in combination with abnormal spontaneous activity including positive sharp waves (PSWs), fibrillations, and/or fasciculation potentials (FP). ${ }^{3}$ The most recent consensus update of the El Escorial Criteria (discussed below) assigned equivalent clinical significance to FP, PSWs, and fibrillation potentials. ${ }^{3}$

\section{Fasciculation potentials}

Fasciculation potentials have long been associated with ALS, and although it is recognized that some patients do not experience FP, the vast majority do. ${ }^{7} \mathrm{FP}$ are irregularly firing spontaneous potentials with morphology similar to a MUP. Although FP are common in ALS, they are not specific for the disease and can be found in patients without MND. ${ }^{26-28}$ Their clinical significance is of greater concern in the presence of unexplained muscle weakness and evidence of chronic neurogenic changes on EMG. ${ }^{22}$

In the evaluation of the patient suspected of having a MND, the EMG evaluation requires that the electromyographer assess for the presence of FP, particularly in muscles that lack PSW and fibrillation potentials. ${ }^{3}$ Intramuscular placement of the needle should be confirmed by volitional muscle activation and then the patient should be instructed to relax the muscle while the needle is left unsupported by the electromyographer. ${ }^{26}$ Needle movement can trigger FP but these potentials have not yet been recognized to have pathologic significance. 
The firing rate or interfasciculation interval of FP vary, requiring observation times of up to 90 seconds to confirm their absence. ${ }^{26}$ There is some evidence to suggest that FP are more frequently observed in proximal muscles as opposed to fibrillation potentials and PSW, which in one study were more frequently identified in distal muscles of patients subsequently diagnosed with ALS. ${ }^{27}$

Due to the shift in clinical importance of FP in the diagnosis of ALS, recent research has focused on defining their characteristics. The origin of fasciculation potentials is unclear; however, there is evidence that they arise both distally in the peripheral axon and from the cell body. ${ }^{28-30}$ de Carvalho and colleagues, in their 1998 paper examining FP in ALS patients and a group of patients with fasciculations from other causes, including three patients with benign fasciculation syndrome, concluded that variations in fasciculation morphology had clinical significance. ${ }^{31}$ They defined both complex (having increased phases) and simple fasciculation potentials and assigned greater clinical significance to complex FP morphology. However, recent studies comparing the EMG characteristics of ALS patients to those with benign fasciculation syndrome have questioned the validity of this classification system. ${ }^{27,32}$ According to EMG results of a combined 35 ALS patients and 18 diagnosed with benign fasciculation syndrome, no significant differences were seen between the characteristics of the morphology of the FP; both demonstrated simple and complex FP. ${ }^{27,32}$ However, as atrophy and weakness progressed in the ALS subjects, the interfasciculation interval, FP duration, and FP area decreased. ${ }^{27}$

\section{Other Needle EMG Considerations}

To diagnose definite ALS, LMN abnormalities must be documented in at least three of the four anatomic regions ${ }^{2,3}$ and thus the electrodiagnostic study often requires needle examination in each region. Electromyographers routinely study the muscles of the cervical and lumbar regions but may not feel comfortable designing a study that includes muscles innervated by the cranial nerves and nerves of the thoracic region. Muscles innervated by the cranial nerves, such as facial, genioglossus, and sternocleidomastoid, may be needed to identify bulbar dysfunction and confirm presence of disease above the level of the cervical spine (Figure 1). ${ }^{33}$ The trapezius is another muscle often recommended for sampling; however, in addition to supply from the accessory nerve, both the trapezius and sternocleidomastoid receive innervation from the upper cervical roots, which may confound results in the patient with high cervical degenerative changes. ${ }^{34}$ In addition, relaxation may be hard to achieve in these muscles, making interpretation difficult.

The needle evaluation should also include thoracic level muscles, for example by studying the thoracic paraspinal muscles. ${ }^{35}$ Sampling muscles between the T6 -T10 root levels helps to avoid confounding results due to multilevel root innervation from the lower cervical or upper lumbar segments (Figure 2). Other thoracic muscles that could be studied include the rectus abdominus and external oblique (Figure 2). ${ }^{36}$ Involvement of muscles of the thoracic region or muscles of the neck in ALS increases the likelihood of impaired respiratory function and should be reported as such in the consultation report. ${ }^{34-36}$

Evidence of involvement in additional body regions is used to meet the El Escorial criteria for the diagnosis of ALS. To reduce patient discomfort and anxiety, the number of muscles 
examined should be minimized, but sufficient to reach the correct diagnosis. The needle examination should begin with the most severely involved limb, looking for abnormalities in at least two muscles with different innervations. ${ }^{1,6}$ The examination then progresses to another anatomic region with sampling of distal and proximal muscles, preferably in a limb with strength abnormalities. Thoracic and bulbar muscles should then be sampled. When neurogenic changes have been identified in three anatomic regions, the needle EMG examination can be concluded. If abnormalities are not identified in any one region but suspicion for a MND remains high, the needle evaluation must expand and may ultimately include examination of muscles in all four regions.

\section{Repetitive Stimulation, Single Fiber, and Other Specialized Studies in ALS}

Repetitive stimulation and single fiber EMG are also often abnormal in patients with ALS. It is well documented that instability of neuromuscular transmission in ALS results from collateral nerve terminal sprouting. Abnormalities on repetitive stimulation in ALS patients were first reported by Mulder and colleagues in 1959 and subsequently have been confirmed. ${ }^{37-39}$ Similar to abnormalities seen in myasthenia gravis, decrements with slow repetitive stimulation, maximal decrement after 3-5 stimuli, and repair after brief exercise have been observed in ALS. ${ }^{6}$ In a recent study $34.4 \%$ of 32 median nerves of ALS patients revealed a drop of greater than $10 \%$ in CMAP amplitude. ${ }^{40}$ In addition, results from single fiber EMG parallel those seen on repetitive stimulation. Abnormalities of jitter, blocking, and fiber density correlate positively with increased muscle atrophy and weakness, likely reflecting both the presence of immature sprouting of nerve terminals and the extent of reinnervation. ${ }^{41}$ Whether these electrodiagnostic strategies will ultimately provide meaningful diagnostic information in cases of ALS remains to be determined, but should not deter diagnosis if all other clinical and electrodiagnostic evidence is consistent.

Motor unit number estimation (MUNE) provides the means to estimate the number of motor units and thus the number of LMNs innervating a muscle. ${ }^{42}$ MUNE is determined by measuring the size of the maximum CMAP amplitude and dividing it by the size of the average surface-detected MUP, providing an estimate of the number of motor units in a muscle. ${ }^{43}$ This number can be followed over time, documenting motor neuron loss and subsequent sprouting of remaining neurons leading to enlarged motor units. There are multiple methods to estimate motor unit number. MUNE has demonstrated that reinnervation by collateral sprouting can prevent reduction in strength and CMAP amplitude despite loss of up to half the motor units in MND. ${ }^{44}$ MUNE has been validated as an outcome measure for ALS clinical trials given its strength in quantifying changes from motor unit loss earlier in the disease, prior to changes in CMAP or muscle strength.

\section{El Escorial and Awaji-shima Criteria}

\section{Revised El Escorial Criteria}

The El Escorial Criteria were initially developed in 1990 by the World Federation of Neurology to standardize the diagnosis of ALS and ensure inclusion of homogeneous patient populations in clinical trials. The criteria were revised in 1998 (Airlie House) and more recently in 2008 (Awaji-shima). ${ }^{2,3}$ These criteria divide the motor system into 4 anatomic 
regions: bulbar, cervical, thoracic, and lumbosacral. Clinical evidence of UMN and LMN pathology is sought within each region; the certainty of diagnosis depends on how many regions reveal UMN and/or LMN pathology. Five categories of certainty for the diagnosis were defined including: clinically definite, clinically probable, clinically probable with laboratory support, clinically possible, and clinically suspected ALS. ${ }^{2}$ For a diagnosis of clinically definite ALS, clinical evidence alone by the presence of both UMN and LMN signs was required in three of the four anatomic regions. ${ }^{2}$ However, the revised El Escorial criteria have been criticized for being too restrictive. In a population-based study, Traynor et al. found that $10 \%$ of 254 patients who died from ALS did not fulfill criteria beyond possible ALS, never reaching eligibility for clinical trial participation. ${ }^{45}$

\section{Awaji-shima update}

To address the restrictive nature of the El Escorial criteria, an international symposium was held in Awaji-shima Japan in December of 2006 with the intent of improving the speed and certainty of diagnosis. ${ }^{3}$ The recommendations from the symposium were published in 2008. These criteria classify the certainty level of the diagnosis of ALS into one of three categories: clinically definite, probable, and possible (Figure 3). The category of "Laboratory Supported Probable ALS" became unnecessary based on the consensus panel's decision that clinical features of neurogenic change and neurogenic EMG findings should have the same diagnostic significance in an individual muscle, and can be considered together in a single limb to meet the required abnormalities for diagnosis of ALS. In addition, FP were recognized as evidence of acute denervation and given equal significance to fibrillation and PSW potentials. The dialogue continues in the literature regarding the effect these changes have had on the timeliness of reaching diagnostic certainty; reports have been conflicting. ${ }^{46,47}$

\section{Other Considerations}

Will Genetic Testing or Magnetic Resonance Imaging ever Replace Electrodiagnostic Testing (EDx) in the Workup of Amyotrophic Lateral Sclerosis or other Motor Neuron Diseases?

The past decade has brought remarkable advances in our understanding of the genetic defects underlying many forms of motor neuron diseases, not only spinal muscular atrophy and familial forms of ALS (FALS) but even sporadic ALS. Certainly in SMA and FALS, the causative genes responsible for these diseases have been localized to specific regions of various chromosomes. However, what molecular testing or magnetic resonance imaging (MRI) does not reveal is the physiological functioning of the nerves and muscles; only electrodiagnostic testing identifies how the neuromuscular system is working. Thus the electrodiagnostic evaluation is not only useful in diagnosis, but is also helpful in monitoring progression of the disease, response to interventions, and in determining prognosis. ${ }^{48}$

\section{Clinical Pearls}

- In patients suspected to have a motor neuron disease, clinicians should perform a thorough electrodiagnostic evaluation, including peripheral nerve conduction 
studies (NCS) and needle electromyography (EMG) to both exclude treatable disease and gather evidence toward a diagnosis of amyotrophic lateral sclerosis (ALS). Because prognosis and survival can be vastly different among the motor neuron disorders, differentiating among these disorders and making an accurate and timely diagnosis are paramount. With early motor neuron disease, LMN abnormalities may be hidden from clinical appreciation and electrodiagnostic evaluation becomes extremely valuable.

- $\quad$ MND studies allow the exclusion of treatable neuropathies, such as multifocal motor neuropathy, from the differential diagnosis and should include proximal stimulation sites to rule out conduction block and temporal dispersion, which are not typical findings in ALS.

- The discovery of sensory abnormalities, while suggestive of a different disease process, do not completely exclude ALS.

- Neuromuscular junction abnormalities are often identified in patients with ALS and should not dissuade the diagnosis if other clinical and electrodiagnostic features point to MND. It is well documented that instability of neuromuscular transmission in ALS results from collateral nerve terminal sprouting.

- The needle EMG is the most important study in determining diagnostic certainty of ALS. Acute (denervation evidence via presence of PSW, fibrillations, and FPs) and chronic neurogenic changes (as evidenced by decreased MUP recruitment with rapidly firing and reduced numbers of MUPs, as well as large amplitude, long duration MUP) is required for diagnosis of ALS. \#

- Although FP are common in ALS, they are not specific for the disease and can be found in patients without MND. The presence of fasciculation potentials is more ominous of motor neuron disease if it is accompanied by unexplained muscle weakness, evidence of chronic neurogenic changes on EMG, and seen in proximal muscles. The recent changes in the revised El Escorial criteria, recommended by the Awaji-shima consensus group, have increased the diagnostic significance of fasciculation potentials to equal that of fibrillation and positive sharp wave potentials in the needle EMG examination of the patient suspected of having ALS.\#

- Needle electromyography of a proximal muscle, such as the genioglossus, may be needed to identify bulbar dysfunction. Both the trapezius and sternocleidomastoid, which receive dual innervation from cranial nerves and upper cervical roots, may be confounded in patients with cervical degenerative changes.

\section{References}

1. Krivickas LS. Amyotrophic lateral sclerosis and other motor neuron diseases. Phys Med Rehabil Clin N Am. 2003 May; 14(2):327-45. [PubMed: 12795519]

2. Brooks BR. El Escorial World Federation of Neurology criteria for the diagnosis of amyotrophic lateral sclerosis. Subcommittee on Motor Neuron Diseases/Amyotrophic Lateral Sclerosis of the World Federation of Neurology Research Group on Neuromuscular Diseases and the El Escorial "Clinical limits of amyotrophic lateral sclerosis" workshop contributors. J Neurol Sci. 1994 Jul; 124(Suppl):96-107. [PubMed: 7807156] 
3. de Carvalho M, Dengler R, Eisen A, England JD, Kaji R, Kimura J, Mills K, Mitsumoto H, Nodera H, Shefner J, Swash M. Electrodiagnostic criteria for diagnosis of ALS. Clin Neurophysiol. 2008 Mar; 119(3):497-503. [PubMed: 18164242]

4. Ragonese P, Cellura E, Aridon P, D'amelio M, Spataro R, Taiello AC, Maimone D, La Bella V, Savettieri G. Incidence of amyotrophic lateral sclerosis in Sicily: A population based study. Amyotroph Lateral Scler. 2012 May; 13(3):284-287. [PubMed: 22409362]

5. Chiò A, Calvo A, Moglia C, Mazzini L, Mora G. PARALS study group. Phenotypic heterogeneity of amyotrophic lateral sclerosis: a population based study. J Neurol Neurosurg Psychiatry. 2011 Jul; 82(7):740-746. [PubMed: 21402743]

6. Daube JR. Electrodiagnostic studies in amyotrophic lateral sclerosis and other motor neuron disorders. Muscle. 2000 Oct; 23(10):1488-1502.

7. Inghilleri M, Iacovelli E. Clinical neurophysiology in ALS. Arch Ital Biol. 2011 Mar; 149(1):57-63. [PubMed: 21412716]

8. Argyriou AA, Polychronopoulos P, Talelli P, Chroni E. F wave study in amyotrophic lateral sclerosis: assessment of balance between upper and lower motor neuron involvement. Clin Neurophysiol. 2006 Jun; 117(6):1260-1265. [PubMed: 16678483]

9. de Carvalho M, Swash M. Nerve conduction studies in amyotrophic lateral sclerosis. Muscle Nerve. 2000 Mar; 23(3):344-352. [PubMed: 10679710]

10. Mills KR, Nithi KA. Peripheral and central motor conduction in amyotrophic lateral sclerosis. J Neurol Sci. 1998 Jul 15; 159(1):82-87. [PubMed: 9700708]

11. Ikeda M, Oka Y. The relationship between nerve conduction velocity and fiber morphology during peripheral nerve regeneration. Brain Behav. 2012 Jul; 2(4):382-90. [PubMed: 22950042]

12. Gregory R, Mills K, Donaghy M. Progressive sensory nerve dysfunction in amyotrophic lateral sclerosis: a prospective clinical and neurophysiological study. J Neurol. 1993 May; 240(5):309_ 14. [PubMed: 8326338]

13. Mondelli M, Rossi A, Passero S, Guazzi GC. Involvement of peripheral sensory fibers in amyotrophic lateral sclerosis: electrophysiological study of 64 cases. Muscle Nerve. 1993 Feb; 16(2):166-72. [PubMed: 8429840]

14. Heads T, Pollock M, Robertson A, Sutherland WH, Allpress S. Sensory nerve pathology in amyotrophic lateral sclerosis. Acta Neuropathol. 1991; 82(4):316-20. [PubMed: 1662002]

15. Pugdahl K, Fuglsang-Frederiksen A, de Carvalho M, Johnsen B, Fawcett PR, Labarre-Vila A, Liguori R, Nix WA, Schofield IS. Generalised sensory system abnormalities in amyotrophic lateral sclerosis: a European multicentre study. J Neurol Neurosurg Psychiatry. 2007 Jul; 78(7): 746-9. [PubMed: 17575020]

16. Guo YS, Wu DX, Wu HR, Wu SY, Yang C, Li B, Bu H, Zhang YS, Li CY. Sensory involvement in the SOD1-G93A mouse model of amyotrophic lateral sclerosis. Exp Mol Med. 2009 Mar 31; 41(3):140-150. [PubMed: 19293633]

17. Pugdahl K, Fuglsang-Frederiksen A, Johnsen B, de Carvalho M, Fawcett PR, Labarre-Vila A, Liguori R, Nix WA, Schofield IS. A prospective multicentre study on sural nerve action potentials in ALS. Clin Neurophysiol. 2008 May; 119(5):1106-10. Epub 2008 Mar 4. [PubMed: 18313354]

18. Kouyoumdjian JA, Morita Mda P, Araújo RG. X-linked spinal and bulbar muscular atrophy (Kennedy's disease) with long-term electrophysiological evaluation: case report. Arq Neuropsiquiatr. 2005 Mar; 63(1):154-159. [PubMed: 15830083]

19. Al-Maawali A, Rolfs A, Klingenhaeger M, Yoon G. Hereditary spastic paraplegia associated with axonal neuropathy: a novel mutation of SPG3A in a large family. J Clin Neuromuscul Dis. 2011 Mar; 12(3):143-6. [PubMed: 21321493]

20. Chroni E, Howard RS, Spencer GT, Panayiotopoulos CP. Motor nerve conduction velocities calculated by $\mathrm{F}$ tacheodispersion in patients with anterior horn diseases. Electromyogr Clin Neurophysiol. 1996 Jun; 36(4):199-205. [PubMed: 8803491]

21. de Carvalho M, Scotto M, Lopes A, Swash M. F-Waves and the corticospinal lesion in amyotrophic lateral sclerosis. Amyotroph Lateral Scler Other Motor Neuron Disord. 2002 Sep; 3(3):131-136. [PubMed: 12495574] 
22. de Carvalho M. Pathophysiological significance of fasciculations in the early diagnosis of ALS. Amyotroph Lateral Scler Other Motor Neuron Disord. 2000 Mar; 1(Suppl 1):S43-46. [PubMed: 11464925]

23. Falck B, Alaranta H. Fibrillation potentials, positive sharp waves and fasciculation in the intrinsic muscles of the foot in healthy subjects. J Neurol Neurosurg Psychiatry. 1983 Jul; 46(7):681-683. [PubMed: 6886709]

24. Burch J, Warren-Gash C, Ingham V, Patel M, Bennett D, Chaudhuri KR. Myasthenia gravis--a rare presentation with tongue atrophy and fasciculation. Age Ageing. 2006 Jan; 35(1):87-8. [PubMed: $16364941]$

25. Whitaker $\mathrm{CH}$, Malchoff CD, Felice KJ. Treatable lower motor neuron disease due to vitamin D deficiency and secondary hyperparathyroidism. Amyotroph Lateral Scler Other Motor Neuron Disord. 2000 Sep; 1(4):283-286. [PubMed: 11465022]

26. Mills KR. Detecting fasciculations in amyotrophic lateral sclerosis: duration of observation required. J Neurol Neurosurg Psychiatry. 2011 May; 82(5):549-551. [PubMed: 20462913]

27. Mills KR. Characteristics of fasciculations in amyotrophic lateral sclerosis and the benign fasciculation syndrome. Brain. 2010 Nov; 133(11):3458-3469. [PubMed: 20959307]

28. Kleine BU, Stegeman DF, Schelhaas HJ, Zwarts MJ. Firing pattern of fasciculations in ALS: evidence for axonal and neuronal origin. Neurology. 2008 Jan 29; 70(5):353-359. [PubMed: 18227416]

29. Kanai K, Kuwabara S, Misawa S, Tamura N, Ogawara K, Nakata M, Sawai S, Hattori T, Bostock H. Altered axonal excitability properties in amyotrophic lateral sclerosis: impaired potassium channel function related to disease stage. Brain. 2006 Apr; 129(Pt 4):953-962. [PubMed: 16467388]

30. Nakata M, Kuwabara S, Kanai K, Misawa S, Tamura N, Sawai S, Hattori T, Bostock H. Distal excitability changes in motor axons in amyotrophic lateral sclerosis. Clin Neurophysiol. 2006 Jul; 117(7):1444-1448. [PubMed: 16765084]

31. de Carvalho M, Swash M. Fasciculation potentials: a study of amyotrophic lateral sclerosis and other neurogenic disorders. Muscle Nerve. 1998 Mar; 21(3):336-344. [PubMed: 9486862]

32. Kleine BU, Boekestein WA, Arts IM, Zwarts MJ, Schelhaas HJ, Stegeman DF. Fasciculations and their F-response revisited: high-density surface EMG in ALS and benign fasciculations. Clin Neurophysiol. 2012 Feb; 123(2):399-405. [PubMed: 21820354]

33. Pinto S, de Carvalho M. Motor responses of the sternocleidomastoid muscle in patients with amyotrophic lateral sclerosis. Muscle Nerve. 2008 Oct; 38(4):1312-1317. [PubMed: 18785186]

34. Tubbs RS, Shoja MM, Loukas M, Lancaster J, Mortazavi MM, Hattab EM, Cohen-Gadol AA. Study of the cervical plexus innervation of the trapezius muscle. J Neurosurg Spine. 2011 May; 14(5):626-629. [PubMed: 21388290]

35. de Carvalho M, Pinto S, Swash M. Motor unit changes in thoracic paraspinal muscles in amyotrophic lateral sclerosis. Muscle Nerve. 2009 Jan; 39(1):83-86. [PubMed: 19086064]

36. Xu Y, Zheng J, Zhang S, Kang D, Zhang J, Fan D. Needle electromyography of the rectus abdominis in patients with amyotrophic lateral sclerosis. Muscle Nerve. 2007 Mar; 35(3):383-385. [PubMed: 17034039]

37. Mulder DW, Lambert EH, Eaton LM. Myasthenic syndrome in patients with amyotrophic lateral sclerosis. Neurology. 1959; 9:627-631. [PubMed: 14425103]

38. Wang FC, De Pasqua V, Gerard P, Delwaide PJ. Prognostic value of decremental responses to repetitive nerve stimulation in ALS patients. Neurology. 2001; 57:897-899. [PubMed: 11552026]

39. Henderson R, Baumann F, Hutchinson N, McCombe P. CMAP decrement in ALS. Muscle Nerve. 2009; 39:555-556. [PubMed: 19296459]

40. Yamashita S, Sakaguchi H, Mori A, Kimura E, Maeda Y, Hirano T, Uchino M. Significant CMAP decrement by repetitive nerve stimulation is more frequent in median than ulnar nerves of patients with amyotrophic lateral sclerosis. Muscle Nerve. 2012 Mar; 45(3):426-8.10.1002/mus.22301 [PubMed: 22334178]

41. Cui LY, Liu MS, Tang XF. Single fiber electromyography in 78 patients with amyotrophic lateral sclerosis. Chin Med J (Engl). 2004 Dec; 117(12):1830-3. [PubMed: 15603713] 
42. Lomen-Hoerth C, Slawnych MP. Statistical motor unit number estimation: from theory to practice. Muscle Nerve. 2003 Sep; 28(3):263-272. [PubMed: 12929186]

43. Rashidipour O, Chan KM. Motor unit number estimation in neuromuscular disease. Can J Neurol Sci. 2008 May; 35(2):153-159. [PubMed: 18574927]

44. Daube RJ. Electrophysiologic studies in diagnosis and prognosis of motor neuron diseases. Neurol Clin. 1985; 3:473-493. [PubMed: 3900681]

45. Traynor BJ, Codd MB, Corr B, Forde C, Frost E, Hardiman OM. Clinical features of amyotrophic lateral sclerosis according to the El Escorial and Airlie House diagnostic criteria: A populationbased study. Arch Neurol. 2000 Aug; 57(8):1171-1176. [PubMed: 10927797]

46. Higashihara M, Sonoo M, Imafuku I, Fukutake T, Kamakura K, Inoue K, Hatanaka Y, Shimizu T, Tsuji S, Ugawa Y. Fasciculation potentials in amyotrophic lateral sclerosis and the diagnostic yield of the Awaji algorithm. Muscle Nerve. 2012 Feb; 45(2):175-182. [PubMed: 22246871]

47. de Carvalho M. Does Awaji decrease diagnostic yield in ALS? Muscle Nerve. 2012 Jul; 46(1): 142-3. author reply 143-144. [PubMed: 22693005]

48. de Carvalho M, Swash M. Sensitivity of electrophysiological tests for upper and lower motor neuron dysfunction in ALS: a six-month longitudinal study. Muscle Nerve. 2010 Feb; 41(2):208211. [PubMed: 19697379] 

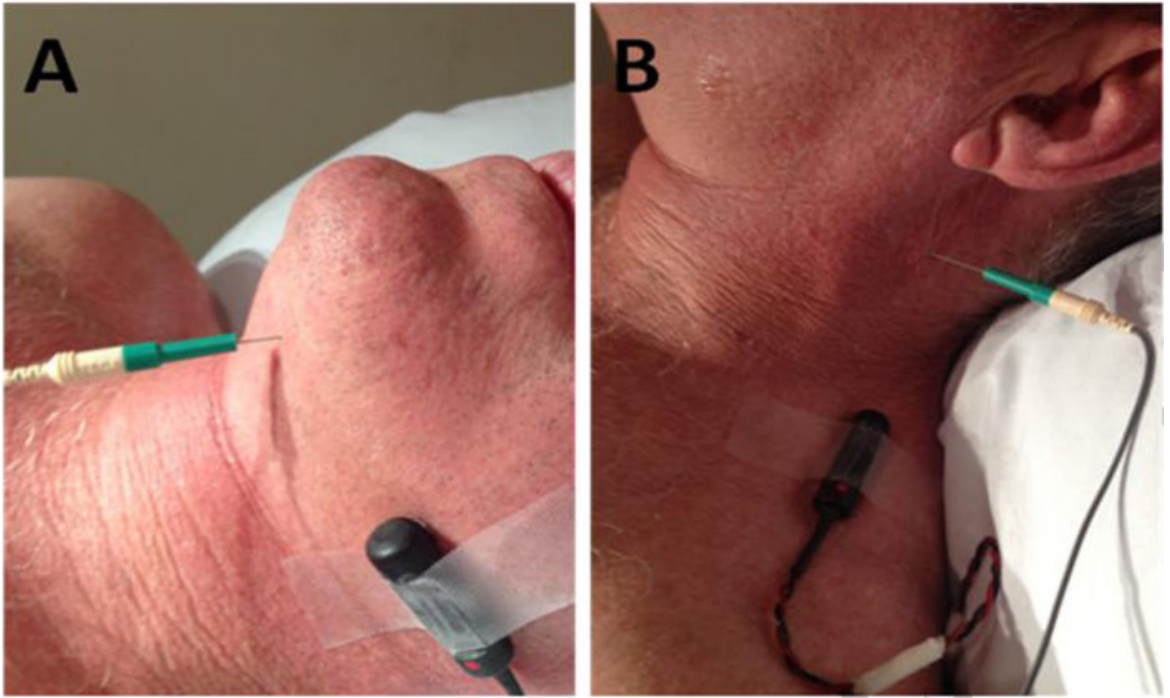

Figure 1.

Needle location for EMG of cranial nerve innervated muscles commonly examined in patients suspected of having amyotrophic lateral sclerosis. A. EMG of the genioglossus muscle. B. EMG of the sternocleidomastoid muscle. 


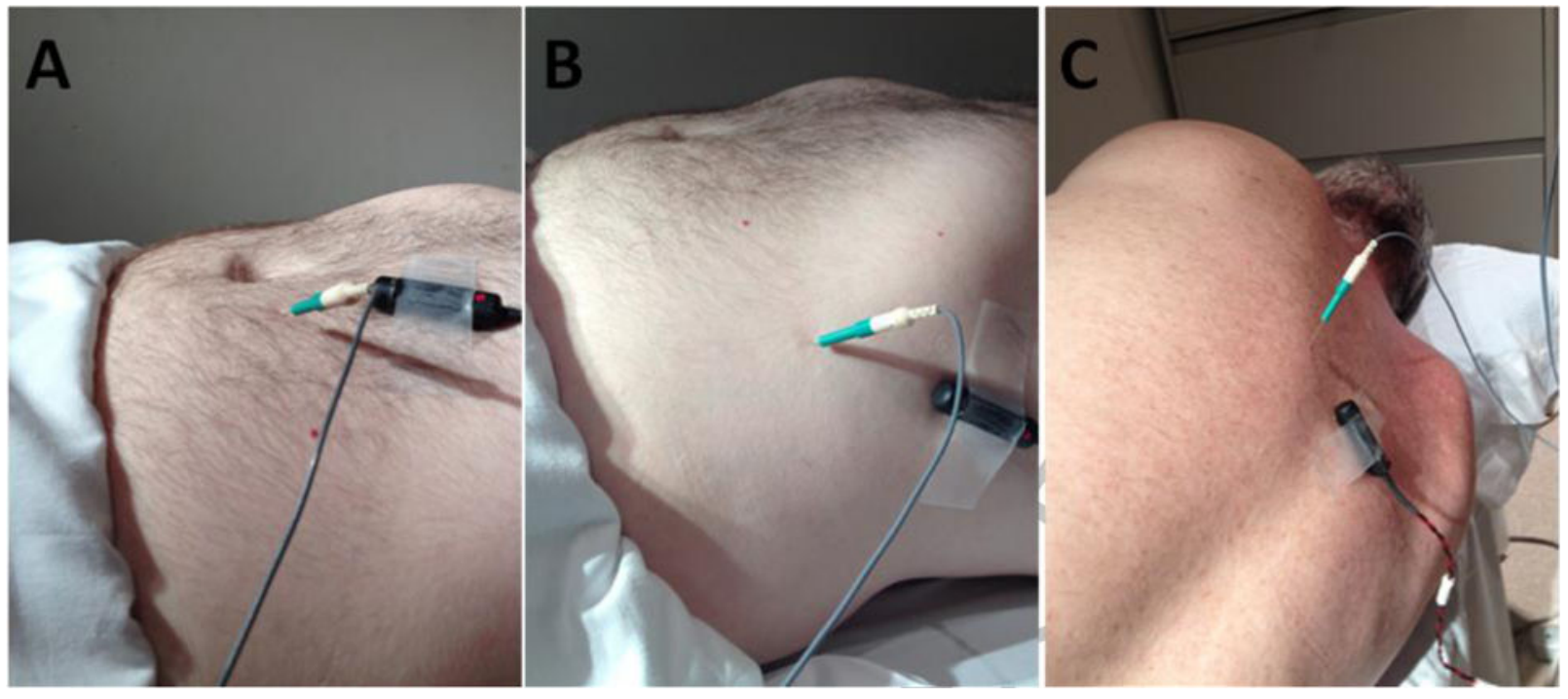

Figure 2.

Needle location for EMG of thoracic innervated muscles. A. EMG study of the rectus abdominis muscle. B. EMG of the external oblique. C. EMG of the thoracic paraspinal muscles at the level of T8, avoiding potential confounding from multi-level innervations of the lower cervical and upper lumbar roots. 
Table 1

Disorders presenting with signs and symptoms that mimic motor neuron disease

Adrenoleukodystrophy
Central nervous system tumors
Cervical and lumbar myelopathy
Chronic inflammatory demyelinating polyradiculoneuropathy
Human Immunodeficiency Virus (HIV)
Inflammatory myopathies
Lambert-Eaton Syndrome
Lyme disease
Multifocal motor neuropathy with conduction block
Multiple Sclerosis
Myasthenia gravis
Polyradiculopathy
Syringomyelia


Table 2

Differential diagnosis of adult motor neuron disease by clinical signs and symptoms

\begin{tabular}{lll}
\hline UMN and LMN & LMN only & UMN only \\
\hline Sporadic ALS & Progressive muscular atrophy & Primary lateral Sclerosis \\
Familial ALS & Spinal muscular atrophy & Hereditary spastic paraplegia \\
& Progressive bulbar palsy & \\
& Monomelic amyotrophy & \\
& Bulbar spinal muscular atrophy & \\
& Poliomyelitis \\
\hline
\end{tabular}

Abbreviations: UMN, upper motor neuron; LMN, lower motor neuron; ALS, amyotrophic lateral sclerosis. 